\title{
Accuracy of the Java Simulation for the Charge Motion in Electric and Magnetic Fields
}

\author{
Masami Morooka ${ }^{1}$ and Midori Morooka ${ }^{2}$ \\ ${ }^{1}$ Department of Electrical Engineering, Fukuoka Inst. Tech, higashi-ku, Fukuoka 811-0295, Japan; \\ ${ }^{2}$ Flash Design Center, Micron Japan, Kamata 5-37-1, Ota-ku, Tokyo144-8721, Japan \\ morooka@fit.ac.jp
}

\begin{abstract}
The accuracy of the Java simulation by the Runge-Kutta method for the charge motion in electric and magnetic fields has been investigated in comparison with the analytical solution. The error of the simulation depends on the time increment, $h$, used for the numerical calculation. If we use an increment that is larger than the boundary value, the simulation results in a non-accurate image of the charge motion. In this case, the simulation almost results in an underestimation, that is, a motion that is smaller than the real motion. The boundary increment is proportional to the mass of the charge, $\mathrm{m}$, and is inversely proportional to the charge, $\mathrm{q}$, and the magnetic field, $\mathrm{B} 0$. The empirical results conclude that the image of the charge motion can be obtained accurately by Java simulation using $h<0.2 \mathrm{~m} / \mathrm{qB}$.

Keywords: Image learning, charge motion in electric and magnetic fields, Java programming, accuracy of Java simulation by Runge-Kutta method.
\end{abstract}

\section{Introduction}

The authors proposed a Java simulation for the rapid and accurate image learning of the charge motion in electric and magnetic fields [1] and for those of the electric characteristics of RCL circuits [2]. In these simulations, the text fields of the selected parameters, such as the electric field and magnetic field for the charge motion or the values of $\mathrm{R}, \mathrm{C}$, and $\mathrm{L}$ for the electric characteristics, are set on the display, and the calculation by the Runge-Kutta method is initiated by clicking the start button after inputting values into the text fields. Immediately following the completion of the calculation, the results are plotted as a figure on the display, e.g. a charge locus for the charge motion or the change of the current and voltage with time for the electric circuit. By changing the values in the text fields, new results can be represented immediately and a simulation under the new condition can be easily obtained. The value of the time increment, $h$, used in the numerical calculation by the Runge-Kutta method is limited to obtain an accurate simulation in spite of the useful simulation.

In this paper, the accuracy of the Java simulation for the charge motion in electric and magnetic fields has been investigated in comparison with the analytical solution, and the boundary value of the time increment to obtain an accurate simulation is shown empirically. 


\section{Numerical Method and Analytical Solutions for the Charge Motion}

\subsection{Equations for the Charge Motion in Electric and Magnetic Fields}

The charge motion in an applied electric field $E=(E x, E y, E z)$ and an applied magnetic field $B=(B x, B y$, $\mathrm{Bz}$ ) is given as

$$
\begin{gathered}
m \frac{d v}{d t}=a v+q E+q v \quad B \quad b\left(\begin{array}{lll}
r & r_{0}
\end{array}\right), \\
\frac{d r}{d t}=\boldsymbol{v} .
\end{gathered}
$$

Here, $t$ is time and $m, q, v=\left(v_{x}, v_{y}, v_{z}\right)$, and $\boldsymbol{r}=(x, y, z)$ are the mass, charge, velocity, and displacement of the charge, respectively. $a$ and $b$ are coefficients of the resistance and restoring forces. $r_{0}$ is the restoring centre. We consider only the electric and magnetic forces, such as the electron motion in a vacuum, to facilitate an easy comparison between the numerical simulation and the analytical solution. We use $\boldsymbol{E}=\left(E_{\mathrm{x}}, 0,0\right), \boldsymbol{B}=\left(0,0, B_{\mathrm{z}}\right), E_{\mathrm{x}}=E_{0} \sin (2 \pi f t)$, and $B_{\mathrm{z}}=B_{0}$ to easily obtain the analytical solution. Here, $E_{0}$ and $B_{0}$ are constants, and $f$ is the frequency of the electric field. In this case, we have four ordinary differential equations from Equations (1) and (2) for the charge motion in the $x-y$ plane.

$$
\begin{gathered}
m \frac{d v_{x}}{d t}=q E_{0} \sin (2 \pi f t)+q v_{y} B_{0^{\prime}} \\
m \frac{d v_{y}}{d t}=q v_{x} B_{0^{\prime}} \\
\frac{d x}{d t}=v_{x^{\prime}} \\
\frac{d y}{d t}=v_{y^{\prime}}
\end{gathered}
$$

\subsection{Numerical Method and Analytical Solutions}

The numerical method for obtaining the solutions of Equations (3) - (6) by Java programming is described in Reference [1] using the fourth-order Runge-Kutta method.

We have the linear differential equation from Equations (3) and (4), as shown below.

$$
\frac{d^{2} v_{x}}{d t}=2 \pi f c E_{0} \cos (2 \pi f t)-c^{2} B_{0}^{2} v_{x}
$$

Here, $c=q / m$. The general solution of Eq. (7) is obtained as the sum of the general solution of the homogeneous equation, $d^{2} v_{x} / d t^{2}+c^{2} B_{0}^{2} v_{x}=0$, and the particular solution.

$$
v_{x}=d_{1} \exp \left(j c B_{0} t\right)+d_{2} \exp \left(-j c B_{0} t\right)+\frac{2 \pi f c E_{0}}{\left(c B_{0}\right)^{2}-(2 \pi f)^{2}} \cos (2 \pi f t) .
$$


Here, $d_{1}$ and $d_{2}$ are unfixed constants and the last term is the particular solution. We use the initial conditions $v_{\mathrm{x}}=v_{0}$ and $v_{\mathrm{y}}=0$ at $t=0$, that is, the charge is injected into the $x$-direction of the fields at the initial velocity $v_{0}$. We obtain the other initial condition, $d v_{\mathrm{x}} / d t=0$ at $t=0$, from Equation (3) using $v_{\mathrm{y}}=0$ at $t=0$. We have the analytical solution, Equation (8), under these initial conditions,

$$
v_{x}=\left\lfloor v_{0}-\frac{2 \pi f c E_{0}}{\left(c B_{0}\right)^{2}-(2 \pi f)^{2}}\right\rfloor \cos \left(c B_{0} t\right)+\frac{2 \pi f c E_{0}}{\left(c B_{0}\right)^{2}-(2 \pi f)^{2}} \cos (2 \pi f t)
$$

We obtain the analytical solution for $v_{y}$ from Equation (3),

$$
v_{y}=\left\lfloor\frac{2 \pi f c E_{0}}{\left(c B_{0}\right)^{2}-(2 \pi f)^{2}}-v_{0}\right\rfloor \sin \left(c B_{0} t\right)-\frac{c B_{0} c E_{0}}{\left(c B_{0}\right)^{2}-(2 \pi f)^{2}} \sin (2 \pi f t
$$

The analytical solutions for $x$ and $y$ under the initial conditions $(x, y)=(0,0)$ at $t=0$ are obtained from Equations (5) and (6),

$$
\begin{aligned}
x= & \frac{1}{c B_{0}}\left\lfloor v_{0}-\frac{2 \pi f c E_{0}}{\left(c B_{0}\right)^{2}-(2 \pi f)^{2}}\right\rfloor \sin \left(c B_{0} t\right)+\frac{c E_{0}}{\left(c B_{0}\right)^{2}-(2 \pi f)^{2}} \sin (2 \pi f t), \\
y & =\frac{1}{c B_{0}}\left\lfloor v_{0}-\frac{2 \pi f c E_{0}}{\left(c B_{0}\right)^{2}-(2 \pi f)^{2}}\right\rfloor \cos \left(c B_{0} t\right)+\frac{1}{2 \pi f} \frac{c B_{0} c E_{0}}{\left(c B_{0}\right)^{2}-(2 \pi f)^{2}} \cos (2 \pi f t) \\
& -\frac{1}{c B_{0}}\left\lfloor v_{0}-\frac{2 \pi f c E_{0}}{\left(c B_{0}\right)^{2}-(2 \pi f)^{2}}\right\rfloor-\frac{1}{2 \pi f} \frac{c B_{0} c E_{0}}{\left(c B_{0}\right)^{2}-(2 \pi f)^{2}} .
\end{aligned}
$$

\section{Comparison of the Numerical Simulations with the Analytical Solutions}

Typical numerical simulations and analytical solutions for an electron motion accelerated by the electric frequency that is synchronized with the Larmor frequency using $E_{0}=30 \mathrm{~V} / \mathrm{m}, f=28.55 \mathrm{MHz}$, and $B_{0}=0.00102 \mathrm{~T}$ are shown in Figure 1. The simulations and solutions are shown in the left and right regions, respectively. The loci of the electron are shown in the upper regions and the changes of $v_{x}$ and $v_{y}$ with time are shown in the lower regions. The numerical calculation is performed using the time increment $h=0.5 \mathrm{~ns}$ by the double precision method. The final position of the calculation at $t=2000 \mathrm{~ns}$, corresponding to 4000 total calculations, is $(x, y)=(0.023912495,0.01770018)$ in meters for the numerical simulation and $(x, y)=(0.023911081,0.017702656)$ for the analytical solution. The differences between the two results are $(\Delta x, \Delta y)=\left(-1.414 \times 10^{-6}, 2.476 \times 10^{-6}\right)$, and their rates are $5.91 \times 10^{-5}$ for $x$ and $1.40 \times 10^{-4}$ for $y$. The differences increase with the increase of the calculation time, such as $(x, y)=(-0.6840279,-0.23154235)$ and $(x, y)=(-0.6834052,-0.23318635)$ after 100,000 calculations in which $(\Delta x, \Delta y)=(0.0006227,-0.001644)$ and their rates are $9.11 \times 10^{-4}$ for $x$ and $7.05 \times 10^{-3}$ for $y$. The global error of this method is $\mathrm{O}\left(h^{4}\right)$ [3]. The position at $t=2000 \mathrm{~ns}$ by the simulation using $h=0.25 \mathrm{~ns}$ is $(x, y)=(0.023911174,0.017702503)$, and their differences from the analytical results are $(\Delta x, \Delta y)=(-9.3$ $\left.x 10^{-8}, 1.53 \times 10^{-7}\right)$, which are approximately $1 / 16$ of the values from the simulation obtained with $h=0.5 \mathrm{~ns}$. 


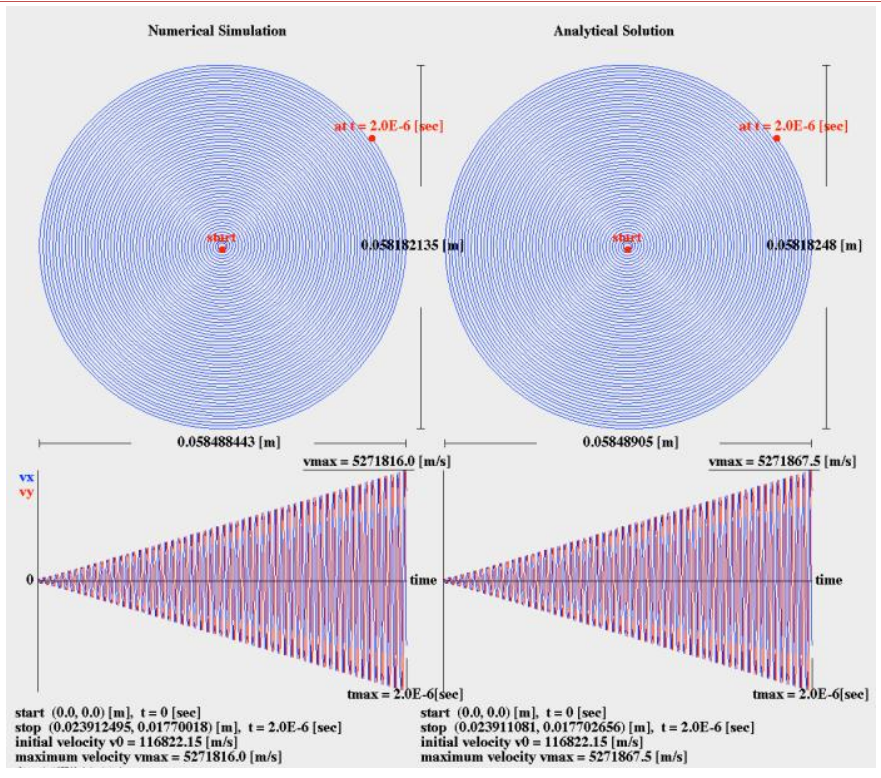

Figure 1: Comparison of a typical simulation with the analytical solution for an electron motion after 4000 calculations, that is, $t=2000 \mathrm{~ns}$, using $\mathrm{h}=0.5 \mathrm{~ns}$ at $\mathrm{EO}=30 \mathrm{~V} / \mathrm{m}, \mathrm{f}=28.55 \mathrm{MHz}$, and BO $=0.00102 \mathrm{~T}$

The error of the Runge-Kutta simulation increases with the increase of $h$, and if we use a large $h$, the Java simulation results in a non-accurate image of the electron motion in comparison with that of the analytical solution, as shown in Figure 2. In this simulation, the range of the electron motion and the velocity at $t=2000 \mathrm{~ns}$ are approximately half that of the accurate analytical values.

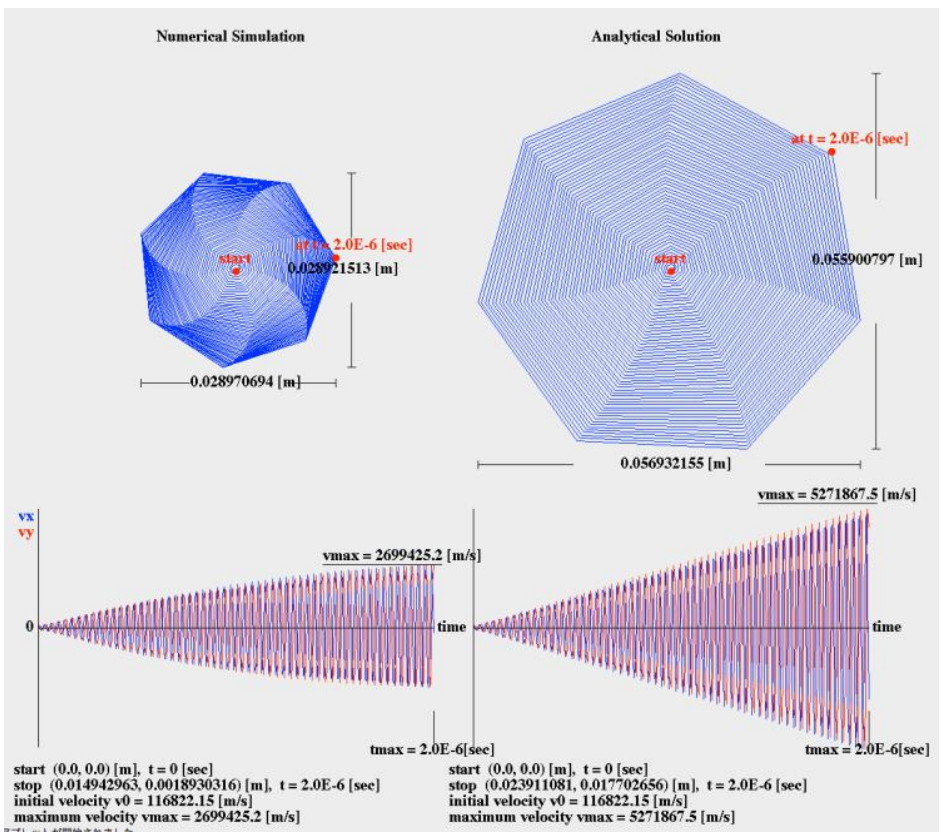

Figure 1: A non-accurate Java simulation for the electron motion using $h=5 \mathrm{~ns}$. The values except $\mathrm{h}$ are the same as those used for the calculations in Figure1. The calculations are performed $\mathbf{4 0 0}$ times, corresponding to the same amount of time, $2000 \mathrm{~ns}$, as in Figure 1.

The accuracy of the Runge-Kutta simulation depends on $h q B_{0} / m$ from Equation (3), and the simulation at $B_{0}=0.0102 \mathrm{~T}$, which is ten times larger than that used in Figure 1 , is not accurate, even with the use of 
$h=0.5 \mathrm{~ns}$, similar to the result from using $h=5 \mathrm{~ns}$ at $B_{0}=0.00102 \mathrm{~T}$, as shown in Figure 3 . We use $f=$ $285.5 \mathrm{MHz}$ for the calculation in Figure 3 to synchronize with the Larmor frequency.

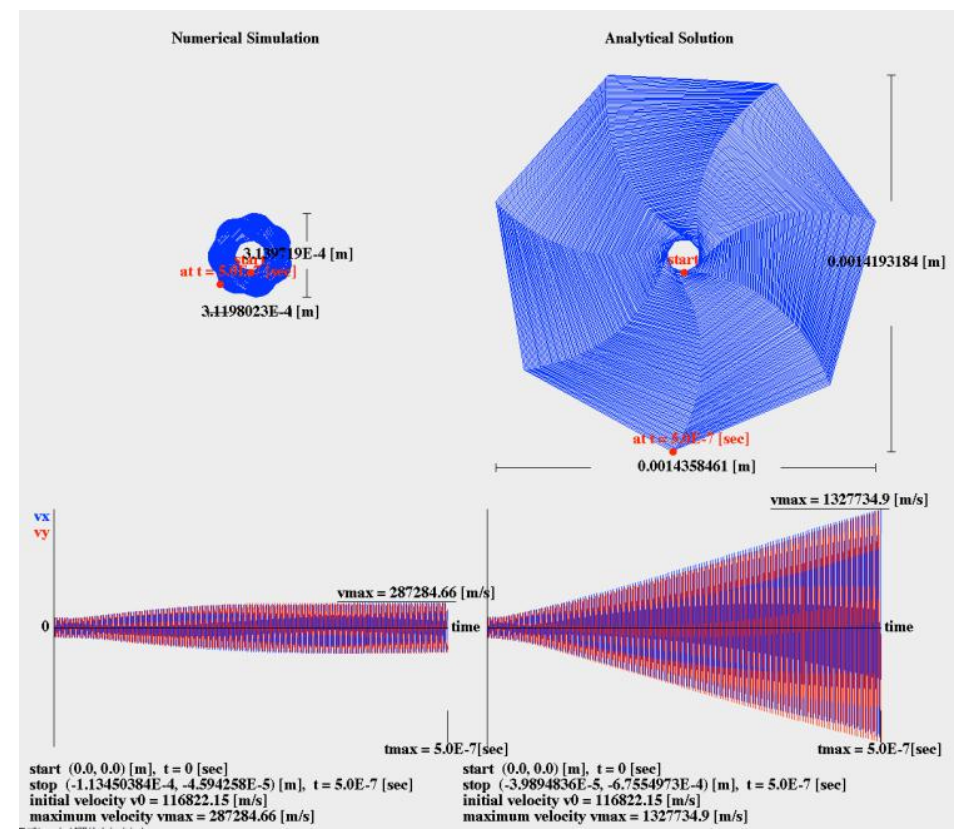

Figure 2: A non-accurate Java simulation for the electron motion even with the use of $h=0.5 \mathrm{~ns}$ due to the use of the ten times larger B0, $0.0102 \mathrm{~T}$, relative to that in Figure $1 . f=285.5 \mathrm{MHz}$ is used to synchronize with the Larmor frequency in this calculation, and the calculations are performed 1000 times until $t=500 \mathrm{~ns}$.

In the case of an ion motion, the value of $h$ used to obtain an accurate image of the motion is not that small, which is in contrast to the electron motion because the accuracy of the Runge-Kutta simulation is proportional to $\mathrm{h} / \mathrm{m}$ from Equation (3). The Java simulations and analytical solutions for an $\mathrm{He}^{+}$motion accelerated by the synchronized electric frequency to the Larmor frequency are shown in Figure 4 using $h=50 \mathrm{~ns}, E_{0}=30 \mathrm{~V} / \mathrm{m}, f=391300 \mathrm{~Hz}$, and $B_{0}=0.102 \mathrm{~T}$. An accurate image for the ion motion can be obtained by the Java simulation even with the use of $h=50 \mathrm{~ns}$. 


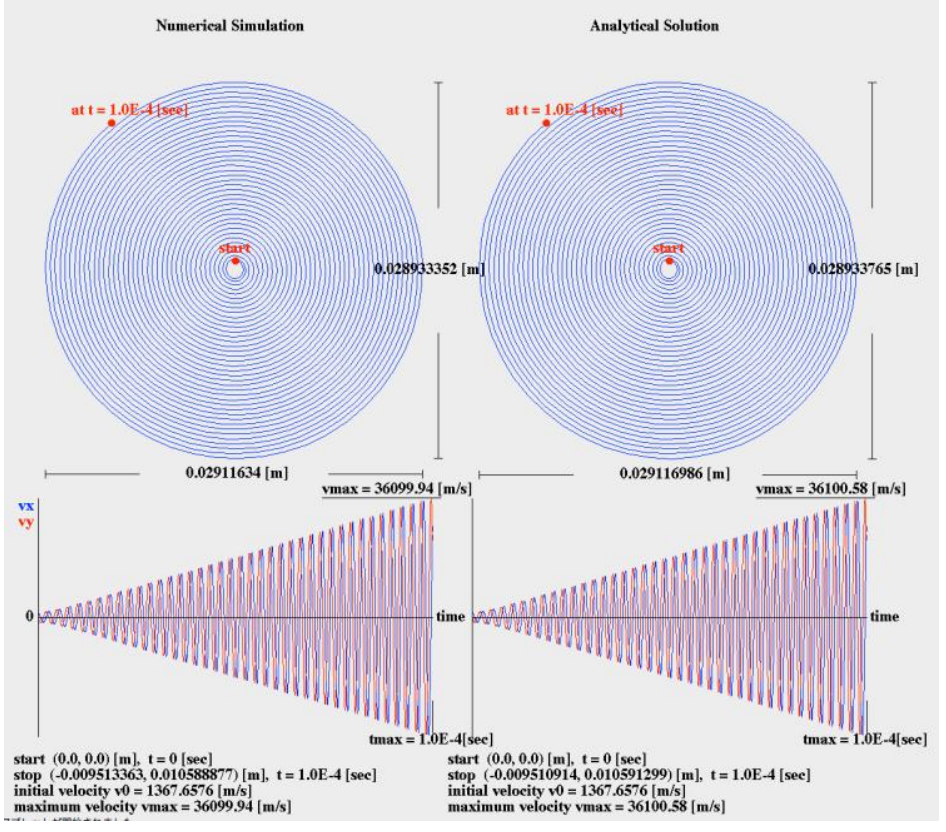

Figure 3: A typical He+ motion accelerated by the electric frequency synchronized with the Larmor frequency using $h=50 \mathrm{~ns}$ at $E 0=30 \mathrm{~V} / \mathrm{m}, f=391300 \mathrm{~Hz}$, and BO $=0.102 \mathrm{~T}$. The calculations are performed 2000 times until $\mathrm{t}$ $=0.0001 \mathrm{sec}$.

\section{Discussion}

The accuracy of the numerical calculation used for the Java simulation depends on $h q B_{0} / m$ from Equation (3). If we use an $h$ that is too large, the calculation is not performed accurately and an appropriate image of the motion is not obtained, as shown in Figures 2 and 3. The accuracy of the simulation is better for evaluating the difference of the motion width, which is $\Delta x_{\max }=x_{\max }-x_{\min }$ or $\Delta y_{\max }$ $=y_{\max }-y_{\min }$, compared to that of the analytical solution. Here, the subscripts max and $\min$ are used to represent the maximum and minimum values, respectively. The values of $\Delta x_{\max }$ at $t=500,1000,2000$, 5000 , and 10000 ns obtained by the simulations and the solutions for the electron motion using $h=0.5$, 1.0, 2.0, 3.0, 4.0, and $5.0 \mathrm{~ns}$ at $E_{0}=30 \mathrm{~V} / \mathrm{m}, f=28.55 \mathrm{MHz}$, and $B_{0}=0.00102 \mathrm{~T}$ are shown in Table 1. The ratio of $\Delta x_{\max }$ obtained by the simulation to that obtained by the solution and the value of $h q B_{0} / \mathrm{m}$ corresponding to each $h$ are also shown in the table. The ratio represents the accuracy of the Java simulation, that is, accuracy = 1 means that the Java simulation is equal to the analytical solution, and an accuracy $>1$ and $<1$ indicate the overestimation and underestimation of the simulations, respectively. The dependence of the accuracy for the electron motion on the value of $h q B_{0} / m$ is shown in Figure 5 . 
Table 1: The motion width, $x \max -x \min$, obtained by the Java simulation and the analytical solution for the electron motion at $t=500,1000,2000,5000$, and $10000 \mathrm{~ns}$ using $\mathrm{h}=\mathbf{0 . 5}, 1.0,2.0,3.0,4.0$, and $5.0 \mathrm{~ns}$ at E0 $=30$

$\mathrm{V} / \mathrm{m}, \mathrm{f}=28.55 \mathrm{MHz}$, and $\mathrm{BO}=0.00102 \mathrm{~T}$

\begin{tabular}{|c|c|c|c|c|c|c|c|}
\hline $\begin{array}{l}\text { Time } \\
\text { (ns) }\end{array}$ & & $\begin{array}{c}h=0.5 \mathrm{~ns} \\
h q B_{0} / m= \\
0.0897\end{array}$ & $\begin{array}{c}h=1.0 \mathrm{~ns} \\
h q B_{0} / m= \\
0.1794\end{array}$ & $\begin{array}{c}h=2.0 \mathrm{~ns} \\
h q B_{0} / m= \\
0.3588\end{array}$ & $\begin{array}{c}h=3.0 \mathrm{~ns} \\
h q B_{0} / m= \\
0.5381\end{array}$ & $\begin{array}{c}h=4.0 \mathrm{~ns} \\
h q B_{0} / m= \\
0.7175\end{array}$ & $\begin{array}{c}h=5.0 \mathrm{~ns} \\
h q B_{0} / m= \\
0.8969\end{array}$ \\
\hline \multirow{3}{*}{500} & & & 9879 & & & 969641 & 567365 \\
\hline & Analytic & 014223453 & 11405 & 014097456 & 027963 & 368473 & 41441 \\
\hline & & & & & & & \\
\hline \multirow{3}{*}{1000} & & & & & & & \\
\hline & Analytical Solution & 0.029138096 & 0.0290887 & 0.028879715 & 0.029069254 & 0.028370567 & 0.028370567 \\
\hline & Ratio (Accuracy) & 9404 & 314 & & & 0.8836217 & 99766 \\
\hline \multirow{3}{*}{2000} & Java S & 443 & 943 & & & 903 & 694 \\
\hline & Analytical Solution & 0.05848905 & 0.05839335 & 0.057914756 & 0.057436377 & 0.057914756 & 32155 \\
\hline & Ratio (Accuracy) & 0.9999896 & & 0.9926698 & 0.9541 & 56373 & 36345 \\
\hline \multirow{3}{*}{5000} & Java Simulation & 0.14653207 & 0.14628382 & 0.14346443 & 0.12560898 & 0.079387136 & 48504 \\
\hline & Analytical Solution & 0.14653541 & 0.14635521 & 0.14584598 & 0.14584598 & 0.14476995 & 0.14280663 \\
\hline & Ratio (Accuracy) & 0.99997723 & 9995122 & 0.9836708 & & 0.5483675 & 0.22747572 \\
\hline \multirow{3}{*}{10000} & Java Simulation & 0.2936057 & 0.29312417 & 0.282346 & 0.21564397 & 0.086871445 & 0.03248504 \\
\hline & Analytical Solution & 0.29361787 & 0.2933971 & & & & \\
\hline & Ratio (Accuracy) & 0.9999585 & 0.99906975 & 0.96233404 & 0.73937434 & 0.29710093 & 0.11489938 \\
\hline
\end{tabular}

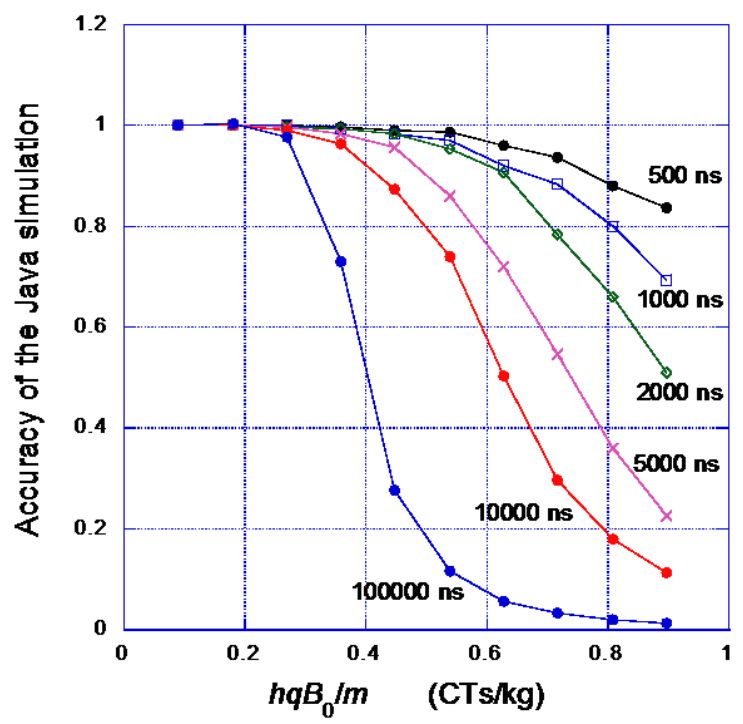

Figure 4: Dependence of the accuracy of the Java simulation on the hqB0/m used for the numerical calculation. Accuracy = 1 means that the Java simulation is equal to the analytical solution. Accuracy $>1$ and $<1$ indicate the overestimation and underestimation of the simulations, respectively.

The values of $\Delta x_{\max }$ at $t=20000,50000,100000,200000$, and 500000 ns obtained by the simulations and the solutions for the $\mathrm{He}^{+}$motion using $h=25,50,100,200,300$, and $400 \mathrm{~ns}$ at $E_{0}=30 \mathrm{~V} / \mathrm{m}, f=$ $391300 \mathrm{~Hz}$, and $B_{0}=0.102 \mathrm{~T}$ are shown in Table 2. The ratio of $\Delta x_{\max }$ and the value of $h q B_{0} / \mathrm{m}$ corresponding to each $h$ are also shown in the table. The dependence of the accuracy for the Java simulation of the $\mathrm{He}^{+}$motion on the value of $h q B_{0} / m$ is shown in Figure 6. 
Masami Morooka and Midori Morooka; Accuracy of the Java Simulation for the Charge Motion in Electric and Magnetic Fields, Transactions on Machine Learning and Artificial Intelligence, Volume 3 No 3 June, (2015);

pp: 15-23

Table 2: The motion width, $x \max -x \min$, obtained by the Java simulation and the analytical solution for the He+ motion at $\mathrm{t}=20000,50000,100000,200000$, and $500000 \mathrm{~ns}$ using $\mathrm{h}=25,50,100,200,300$, and $400 \mathrm{~ns}$ at E0 = 30

$\mathrm{V} / \mathrm{m}, \mathrm{f}=391300 \mathrm{~Hz}$, and $\mathrm{BO}=0.102 \mathrm{~T}$

\begin{tabular}{|c|c|c|c|c|c|c|c|}
\hline $\begin{array}{l}\text { Time } \\
\text { (ns) }\end{array}$ & Methods & $\begin{array}{c}h=25 \mathrm{~ns} \\
h q B_{0} / m= \\
0.0615\end{array}$ & $\begin{array}{c}h=50 \mathrm{~ns} \\
h q B_{0} / m= \\
0.1229\end{array}$ & $\begin{array}{c}h=100 \mathrm{~ns} \\
h q B_{0} / m= \\
0.2459\end{array}$ & $\begin{array}{c}h=200 \mathrm{~ns} \\
h q B_{0} / m= \\
0.4917\end{array}$ & $\begin{array}{c}h=300 \mathrm{~ns} \\
h q B_{0} / m= \\
0.7376\end{array}$ & $\begin{array}{c}h=400 \mathrm{~ns} \\
h q B_{0} / m= \\
0.9834\end{array}$ \\
\hline \multirow{3}{*}{20000} & Java Simulation & 0.0055589615 & 0.0055585667 & $3^{0.005558334}$ & 0.005472003 & 0.005226011 & 0.0047790827 \\
\hline & Analytical Solution & & 0.00555855 & & & & \\
\hline & Ratio ( & & & & & & \\
\hline \multirow{3}{*}{50000} & Java Simulation & 0.01 & & & & & 964 \\
\hline & Analytical Solution & 0.014506063 & 0.01 & & 0.0 & 0.0 & 3999 \\
\hline & Ratio (Ac & 1.00 & & & & & \\
\hline \multirow{3}{*}{$\begin{array}{c}10000 \\
0\end{array}$} & Java Sin & 0.029 & 0.0 & 0.0 & 0.02 & 0.0 & 205 \\
\hline & Analytical Solution & 0.029134804 & 0.029116986 & 0.02899 & 0.028993592 & 0.028 & 5592 \\
\hline & Ratio (Accuracy) & 0.9999998 & 0.999 & 0.9 & 0.97 & 0.83 & 0.5 \\
\hline \multirow{3}{*}{$\begin{array}{c}20000 \\
0\end{array}$} & Java Simulation & 0.05841807 & 0.05838068 & 0.058114525 & 0.055021226 & 0.04045283 & 4748 \\
\hline & Analytical Solution & 0.0584181 & 0.05838337 & 0.058229085 & 0.05778163 & 0.057 & 3163 \\
\hline & Ratio (Accuracy) & 0.999 & 0.999 & & 0.9 & 0.70 & \\
\hline \multirow{3}{*}{500000} & Java Simulation & 0.14663371 & 0.1465667 & 0.14601156 & 0.12891208 & 0.056520145 & 34748 \\
\hline & Analytical Solution & 0.14663388 & 0.14658329 & & 0.14594747 & 0.14540245 & 0.1458892 \\
\hline & Ratio (Accuracy) & 0.99999887 & 0.9998869 & 0.99609965 & 0.88327724 & 0.38871524 & 0.11205408 \\
\hline
\end{tabular}

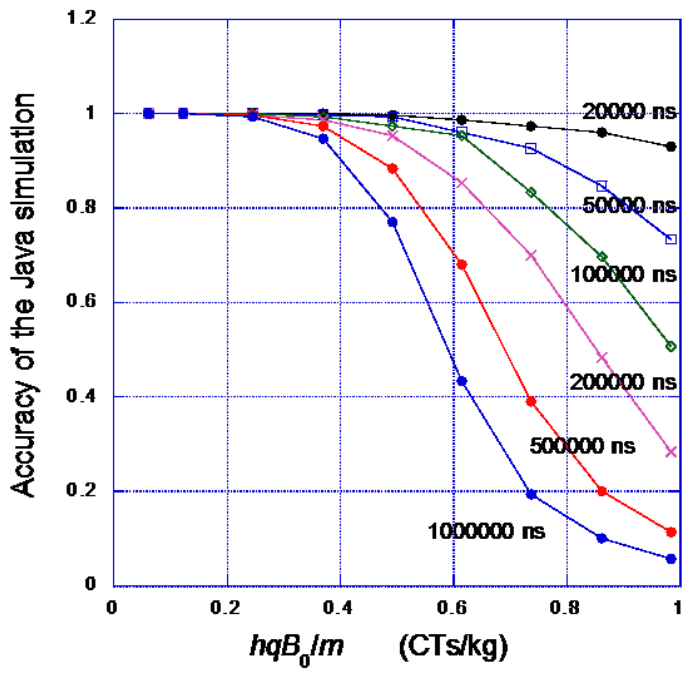

Figure 5: Dependence of the accuracy of the Java simulation for He+ motion on $\mathrm{hqBO} / \mathrm{m}$. Accuracy $=1$ means that the Java simulation is equal to the analytical solution. Accuracy $>1$ and $<1$ indicate the over estimation and under estimation of the simulations, respectively.

\section{Conclusion}

The accuracy of the Java simulation by the Runge-Kutta method for the charge motion in electric and magnetic fields has been investigated in comparison with the analytical solution. The results are summarized as follows: 
1. The accuracy depends on the value of $h q B_{0} / m$ used for the numerical calculation, which also depends on the numerical calculation time.

2. In the case of non-accurate simulation, the simulation almost results in an underestimation, that is, a motion that is smaller than the real motion.

3. An accurate image for the charge motion in electric and magnetic fields is able to be obtained by the Java simulation using a less value of $h q B_{0} / m$ than $0.2 \mathrm{sCT} / \mathrm{kg}$, that is, using $h<0.2 m / q B_{0}$, as shown in Figures 5 and 6.

\section{REFERENCES}

[1]. M. Morooka, S. Qian, and M. Morooka, Image Learnig of Charge Motion in Electric and Magnetic Fields by Java Programming, Transactions on Machine Learnig and Artificial Intelligence, 2014. 2(2): p.1- 19.

[2]. M. Morooka, S. Qian, and M. Morooka, Image Learnig of Electric characteristics of Resistance, Capacitance, Inductance, and their Circuits by Java Programming, Transactions on Machine Learnig and Artificial Intelligence, 2014. 2(3): p.1- 19.

[3]. A. Ralston and C. L. Meek, ENCYCROPEDIA OF COMPUTER SCIENCE, Van Nostrand Rainhold Company (1976), p. 984. 